\title{
Transcranial Direct Current Stimulation in Patients with Prolonged Disorders of Consciousness: Combined Behavioral and Event-Related Potential Evidence
}

\author{
Ye Zhang, Weiqun Song*, Jubao Du, Su Huo, Guixiang Shan and Ran Li \\ Department of Rehabilitation Medicine, Xuan Wu Hospital, Capital Medical University, Beijing, China
}

OPEN ACCESS

Edited by:

Bryan G. Young,

London Health Sciences Centre,

Canada

Reviewed by:

Aldo Ragazzoni,

Fondazione PAS, Italy

Davinia Fernández-Espejo,

University of Birmingham, United

Kingdom

Teneille Emma Gofton,

University of Western Ontario,

Canada

${ }^{*}$ Correspondence:

Weiqun Song

songwq66@126.com

Specialty section:

This article was submitted

to Neurocritical and

Neurohospitalist Care,

a section of the journal

Frontiers in Neurology

Received: 21 August 2017 Accepted: 06 November 2017 Published: 21 November 2017

Citation:

Zhang Y, Song W, Du J, Huo S, Shan G and Li R (2017) Transcranial Direct Current Stimulation in Patients with Prolonged Disorders of

Consciousness: Combined

Behavioral and Event-Related Potential Evidence.

Front. Neurol. 8:620.

doi: 10.3389/fneur.2017.00620
Background: The electrophysiological evidence supporting the therapeutic efficacy of multiple transcranial direct current stimulation (tDCS) sessions on consciousness improvement in patients with prolonged disorders of consciousness (DOCs) has not been firmly established.

Objectives: To assess the effects of repeated tDCS in patients with prolonged DOCs by Coma Recovery Scale-Revised (CRS-R) score and event-related potential (ERP).

Method: Using a sham-controlled randomized double-blind design, 26 patients were randomly assigned to either a real [five vegetative state (VS) and eight minimally conscious state (MCS) patients] or sham (six VS and seven MCS patients) stimulation group. The patients in the real stimulation group underwent 20 anodal tDCS sessions of the left dorsolateral prefrontal cortex (DLPFC) over 10 consecutive working days. The CRS-R score and P300 amplitude and latency in a hierarchical cognitive assessment were recorded to evaluate the consciousness level before tDCS and immediately after the 20 sessions.

Results: The intra-group CRS-R analysis revealed a clinically significant improvement in the MCS patients in the real stimulation group. The inter-group CRS-R analysis showed a significant difference in CRS-R between VS and MCS patients at baseline in both the real and sham stimulation groups. The intra-group ERP analysis revealed a significant increase in P300 amplitude after tDCS in the MCS patients in the real stimulation group, but no significant differences in P300 latency. For the inter-group ERP analysis, we observed significant differences regarding the presence of P300 at baseline between the VS and MCS patients in both groups.

Conclusion: The repeated anodal tDCS of the left DLPFC could produce clinically significant improvements in MCS patients. The observed tDCS-related consciousness improvements might be related to improvements in attention resource allocation (reflected by the P300 amplitude). The findings support the use of tDCS in clinical practice and ERP might serve as an efficient electrophysiological assessment tool in patients with DOCs.

Keywords: transcranial direct current stimulation, coma recovery scale-revised, event-related potentials, P300, disorders of consciousness 


\section{INTRODUCTION}

Patients with severe brain injury may suffer from a wide spectrum of prolonged disorders of consciousness (DOCs), mainly including vegetative state (VS) (1) and minimally conscious state (MCS) (2). Although, some studies have attempted to explore pharmacologic and non-pharmacologic treatment effects, there are currently no evidence-based guidelines on DOCs treatment.

Transcranial direct current stimulation (tDCS) is a form of non-invasive neurostimulation that involves modulating cortical excitability using weak polarizing currents. The dorsolateral prefrontal cortex (DLPFC) is one of the key brain regions of top-down control that has been shown to adjust the course of bottom-up processing through increases in extrastriate neural activity, thereby enhancing attention to stimuli $(3,4)$. Several studies have reported that tDCS of the left DLPFC can transiently improve working memory and cognitive control for emotional information in healthy participants (5-7). Other studies have demonstrated that a single session and multiple sessions of tDCS of the left DLPFC can transiently improve Coma Recovery ScaleRevised (CRS-R) total scores in MCS patients (8-10). Recently, a study used transcranial magnetic stimulation-electroencephalography (TMS-EEG) to assess the electrophysiological effects of tDCS (11). It was found that a single session of tDCS could modulate cortical excitability in patients with DOCs by stimulating the left DLPFC. However, the electrophysiological evidence supporting the therapeutic efficacy of multiple tDCS sessions on consciousness improvement in patients with prolonged DOCs has not been firmly established.

Currently, the most widely accepted tool for assessing consciousness is a behavioral scale. However, because of severe sensory and motor deficits, the lack of behavioral responsiveness of command following is not necessarily indicative of a lack of consciousness. It has been reported that behavioral abilities can fluctuate over time, which would lead to a high rate of misdiagnosis (12). Recently, event-related potential (ERP) recording has been used as an objective and easy evaluation method for assessing cortical information processing capabilities in the absence of overt behavior in patients with DOCs. Some studies have attempted to specifically detect the existence of attentional capabilities by measuring the P300 wave, which is well understood to be a correlate of attention and conscious perception (13-15).

The P300 amplitude mainly depends on stimulus saliency, and it reflects the level of attentional resource allocation (i.e., attentional load), and the P300 latency reflects the speed at which the target stimulus is detected and evaluated (i.e., task complexity) (16-18). In some patients with DOCs, the subject's own name (SON) spoken by a familiar voice (which is an important self-related stimulus) can elicit a stronger cognitive response and activate the cerebral cortex more extensively than non-self-referential emotional stimuli $(15,19)$. Our previous study of patients with DOCs involved using

Abbreviations: $\mathrm{tDCS}$, transcranial direct current stimulation; DOCs, disorders of consciousness; ERP, event-related potential; VS, vegetative state; MCS, minimally conscious state; CRS-R, Coma Recovery Scale-Revised; DLPFC, dorsolateral prefrontal cortex; SON, subject's own name; SDN, subject's derived name; EOG, electrooculography. the SON (spoken by a familiar voice) as the deviant stimulus, and a $1,000 \mathrm{~Hz}$ tone and the subject's derived name (SDN) as the standard stimuli, and we successfully obtained a hierarchical auditory ERP pattern, indicating that the approach could be used as a valuable tool to evaluate patients with DOCs $(15,20,21)$.

Given the significance of ERP to the assessment of electrophysiological changes related to consciousness recovery, we sought to explore ERP and behavioral evidence of consciousness recovery after anodal tDCS of the left DLPFC in VS and MCS patients.

\section{MATERIALS AND METHODS}

\section{Experimental Design}

The study involved a sham-controlled randomized double-blind design, and all enrolled patients were randomly assigned to one of two groups: a real or sham stimulation group. In the real stimulation group, 20 tDCS sessions were administered over 10 consecutive working days (from Monday to Friday) and in the sham stimulation group, 20 sham stimulation sessions were administered over 10 consecutive working days (from Monday to Friday). All the eligible patients underwent behavioral and electrophysiological evaluation by blinded assessors at two time points: (1) baseline (pre-tDCS) and (2) immediately after the 20 active or sham sessions (post-tDCS).

\section{Patients}

Twenty-six patients with severe brain injury (five VS and eight MCS patients in the real stimulation group, and six VS and seven MCS patients in the sham stimulation group) were recruited from the Department of Rehabilitation Medicine at Xuan Wu Hospital, Beijing, China, between July 2015 and June 2017. All the patients were right handed. In the real stimulation group, the etiologies of the patients' conditions were anoxia $(n=2)$, traumatic brain injury $(n=5)$, hemorrhagic stroke $(n=5)$, and ischemic stroke $(n=1)$. In the sham stimulation group, the etiologies were anoxia $(n=3)$, traumatic brain injury $(n=7)$, hemorrhagic stroke $(n=2)$, and ischemic stroke $(n=1)$. The duration of VS or MCS ranged from 1.0 to 17.4 months (mean $5.7 \pm 4.6$ months) in the real stimulation group and 1.4 to 14.6 months (mean $5.0 \pm 3.8$ months) in the sham stimulation group. The general conditions of each of the included patients are shown in Table 1. None of them had a history of neurological disease prior to their coma. All the patients underwent at least one brain computed tomography (CT) or magnetic resonance imaging (MRI) scan. Four patients (VS4, MCS1, MCS2, and MCS5) in the real stimulation group and three (VS4, MCS1, and MCS2) in the sham stimulation group had undergone a left craniotomy to reduce intracranial pressure. None of the patients underwent craniotomy-related plastic surgery. None of them carried a metallic cerebral implant, pacemaker, or neurostimulator. Any other treatments or drugs which modify cortical excitability were excluded. During the 10 days tDCS, all patients received the basic rehabilitation program involving physiotherapy, speech therapy, as well as medical demands during the hospital stay. Written informed consents were acquired from all the patients' families or caregivers. The study was conducted according to the Declaration of Helsinki, and the ethics approval was provided by the ethics committee of the hospital. 


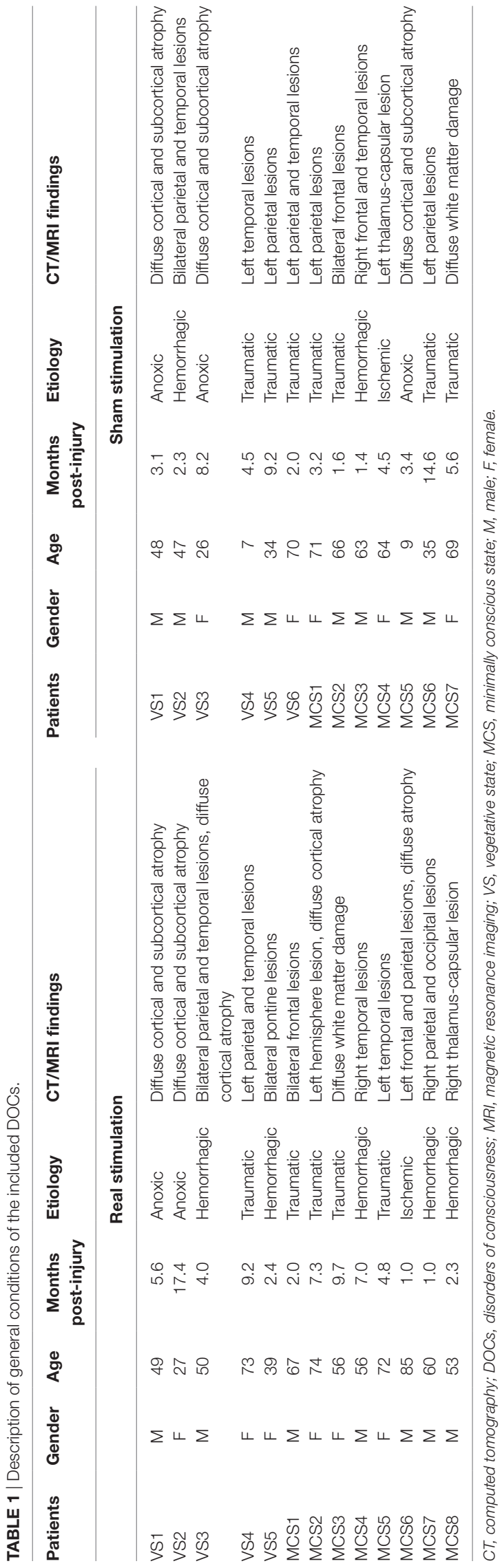

\section{tDCS Protocol}

The tDCS was administered using an Eldith DC-stimulator (neuroConn GmbH, Ilmenau, Germany). A direct current was applied by the battery-driven constant-current stimulator using saline-soaked surface sponge electrodes $(7 \mathrm{~cm} \times 5 \mathrm{~cm})$ with the anode placed over the left DLPFC (position F3 of the 10-20 international electroencephalography system for EEG placement) and the reference cathode placed over the right supraorbital region (approximately position $\mathrm{Fp} 2$ ). The stimulation parameters were slightly modified based on the previous studies $(9,10,22)$. During the real tDCS, the current was increased to $2 \mathrm{~mA}$ from the onset of stimulation. Stimulation lasted $20 \mathrm{~min}$ per session, and it was administered twice a day (one session in the morning and one session in the afternoon) for 10 consecutive working days (from Monday to Friday). For the sham tDCS, the same electrode arrangement and stimulation parameters were employed, except that the stimulator was turned off after $30 \mathrm{~s}$. For the patients who had undergone left craniotomy surgery (VS4, MCS1, MCS2, and MCS5 in the real stimulation group and VS4, MCS1, and MCS2 in the sham stimulation group), the current was increased to $1 \mathrm{~mA}$ instead of $2 \mathrm{~mA}$ from the onset of stimulation.

\section{Behavioral Assessment}

After admission, the patients with DOCs were allowed to familiarize themselves with the ward environment for 3-4 days, and their clinical conditions were allowed to stabilize. Following 1 week of careful daily observation, the diagnoses were made by multiple trained and experienced blinded examiners using the CRS-R. The CRS- $\mathrm{R}$ is a sensitive tool for characterizing the level of consciousness and monitoring neurobehavioral recovery in patients with DOCs. In our study, each patient was assessed by the same two blinded assessors before and after tDCS. They performed separate assessments and then reached a consensus. To ensure patients' best vigilance state, the clinical evaluations were performed at patients' bed in the morning after routine nursing procedures.

\section{ERP Assessment Procedure}

We used two oddball paradigms, both of which have been described previously (15). In the first (Tone-SON paradigm, also called the TO paradigm), a $1,000-\mathrm{Hz}$ tone was used as the standard stimulus, and in the second (SDN-SON paradigm, also called the DO paradigm), the SDN was used as the standard stimulus. SON was used as the deviant stimulus in both paradigms. The $1,000-\mathrm{Hz}$ tone lasted for $100 \mathrm{~ms}$ and was generated using Adobe Audition software (Adobe, Beijing Fistar Technology Ltd. Co., Beijing, China). The SDN was formed by reversing the constituent order of the SON. For each patient, the two-character SON and SDN were recorded by a first-degree family member and digitized for binaural replay (at a maximum sound pressure level of $90 \mathrm{~dB}$ ) during the experiment. The mean durations of the SON and SDN for the VS and MCS patients in the real and sham stimulation groups were not significantly different.

The patients were told that they were going to be played a series of sounds and that they were only required to listen. The stimuli were delivered in a random order, with an interstimulus interval of 0.8-1.2 s. Each paradigm consisted of 500 stimuli, and 
the probability of the deviant stimuli being administered was 0.2 . The order of presentation of the two paradigms was balanced in all four groups of patients. The stimuli were presented using E-prime 2.0 software (Carnegie-Mellon University and University of Pittsburgh, Pittsburgh, PA, USA). The two paradigms lasted approximately $30 \mathrm{~min}$ in total.

\section{ERP Recording}

If the patient's medical stability was adequate, the ERPs were recorded in the ERP experiment room, and if not, the recordings were carried out at the patient's bedside. Patients cleaned their hair before test to decrease the electric resistance of the scalp. ERPs were recorded from midline electrodes $\mathrm{Fz}, \mathrm{Cz}$, and $\mathrm{Pz}$ with 64-channel electrode caps (Neuroscan Inc., Charlotte, NC, USA) according to the international 10-20 system. The reference electrode was placed on the nose, and the ground electrode on the mid forehead. An electrooculogram was acquired using two vertical electrodes placed above and below the left eye, and two horizontal electrodes placed $10 \mathrm{~mm}$ from the lateral canthi of the eyes. The electrode impedance was kept below $5 \mathrm{k} \Omega$ throughout the experiment. The bandpass was $0.1-100 \mathrm{~Hz}$, and the sampling rate was $1,000 \mathrm{~Hz}$. The ERP recording protocol was according to criteria previously published (15). It was performed while the patients were in a wakeful state, with eyes open, and with minimal ambient noise. To ensure the stability of the auditory information processing in the patients with DOCs (18), we recollected the ERP for those with initially low-quality ERP recordings, which potentially reduced false-negative ERP findings.

\section{ERP Analysis}

Electrooculogram artifacts were corrected using the method proposed by Semlitsch et al. (23). Each EEG was split into segments from $100 \mathrm{~ms}$ prestimulus to $700 \mathrm{~ms}$ poststimulus. Then, baseline adjustment was done to ensure that all ERP segments had the same origin. The EEG segment contaminated by amplifier clipping, bursts of electromyography activity, or peak-to-peak deflection exceeding $\pm 100 \mu \mathrm{V}$ were excluded from averaging. The EEG segments were averaged separately for deviant and standard stimuli. The ERP analysis protocol was according to criteria previously published (15). The recognition of P300 waves was performed by trained and experienced blinded assessors. Peak detection was used to obtain the P300 amplitudes and latencies. The P300 amplitude and latency in the patients with DOCs were calculated manually one by one.

\section{Statistical Analysis}

We considered two different types of data: first the behavioral test scores (CRS-R) and second the ERP data (peak P300 latencies from stimulus onset and the P300 amplitudes from the baseline of the stimulus onset). Statistical analysis was performed on the averaged traces from each participant using SPSS version 22.0 (SPSS, Chicago, IL, USA).

Regarding the behavioral data, a repeated measure ANOVA with Stimulation (real, sham) $\times$ Group $(\mathrm{VS}, \mathrm{MCS}) \times$ Time (pretDCS, post-tDCS) mixed ANOVA was performed on CRS-R total scores and CRS-R subscale scores. For the CRS-R total scores at baseline, a two-way ANOVA with Stimulation (real, sham $) \times$ Group (VS, MCS) was performed.

Regarding the ERP data, a repeated measure ANOVA with Stimulation (real, sham) $\times$ Paradigms $(\mathrm{TO}, \mathrm{DO}) \times$ Time $($ pretDCS, post-tDCS) mixed ANOVA was performed on P300 amplitude and latency. A test for post hoc comparisons was used when the results reached significance at $p<0.05$. Multiple comparisons using Bonferroni correction ( $n$ comparisons) had to be performed, and results were considered significant at $p<(0.05 / \mathrm{n})$. Fisher's exact test was used to analyze dichotomous variables indicating the presence or absence of the P300 wave and patient diagnosis (VS or MCS) at baseline. The significance level was set at $p=0.05$. All the data were analyzed using SPSS software.

\section{RESULTS}

\section{Patients' Characteristics}

The real and sham stimulation groups did not differ for important demographics (etiology, age, and time since onset). No adverse effects that were potentially related to tDCS were observed in any of the patients.

\section{CRS-R Results}

In the real and sham stimulation groups, we compared the CRS-R total scores and CRS-R subscale scores at baseline (pre-tDCS) and after 20 sessions of tDCS (post-tDCS) in each group (VS and MCS), and the CRS-R total scores between each group (VS and MCS) at baseline.

Regarding the CRS-R total scores, the Stimulation (real, sham $) \times$ Group $(\mathrm{VS}, \mathrm{MCS}) \times$ Time $($ pre-tDCS, post-tDCS) repeated measures ANOVA revealed a main effect of Time $[F(1,22)=30.48$, $p<0.0005]$, a main effect of Group $[F(1,22)=118.05, p<0.0005]$, an interaction between Time and Group $[F(1,22)=15.90, p=0.001]$, an interaction between Time and Stimulation $[F(1,22)=7.41$, $p=0.012]$, and, most important, a significant three-way interaction $[F(1,22)=8.36, p=0.008]$. Post hoc comparison revealed that after tDCS, a significant improvement was observed in the MCS patients in the real stimulation group $[F(1,22)=72.54, p<0.0005]$, but no significant difference was observed in the VS patients in the real stimulation group or in the sham stimulation group $(F<1)$, nor MCS patients in the sham stimulation group $[F(1,22)=4.21$, $p=0.052]$. The two-way ANOVA analysis at baseline showed a significant main effect of Group $[F(1,22)=70.23, p<0.0005]$, and no significant main effect of Stimulation $[F(1,22)=4.30, p=0.05]$ or interaction between Group and Stimulation $(F<1)$, suggesting a significant difference in CRS-R total scores between the VS and MCS patients at baseline, as shown in Table 2 and Figure $\mathbf{1}$.

For the CRS-R subscale scores, the repeated measures ANOVA and post hoc comparison revealed a significant improvement in the auditory $[F(1,22)=29.97, p<0.0005]$, visual $[F(1,24)=22.96$, $p<0.0005]$, motor $[F(1,22)=54.78, p<0.0005]$, verbal $[F(1$, $22)=77.79, p<0.0005]$, communication $[F(1,22)=36.67$, $p<0.0005]$, and arousal $[F(1,24)=19.91, p<0.0005]$ subscale scores in the MCS patients in the real stimulation group, but no significant difference was observed in the VS patients in the real stimulation group or in the sham stimulation group, as shown in Table 2 and Figure 1. 
TABLE 2 | CRS-R total scores and CRS-R subscale scores in VS and MCS groups at baseline (pre-tDCS) and after 20 sessions of tDCS (post-tDCS).

\begin{tabular}{|c|c|c|c|c|c|}
\hline \multirow[t]{2}{*}{ Real stimulation } & \multicolumn{2}{|c|}{ CRS-R Scores } & \multirow[t]{2}{*}{ Sham stimulation } & \multicolumn{2}{|c|}{ CRS-R scores } \\
\hline & Pre-tDCs & Post-tDCs & & Pre-tDCs & Post-tDCs \\
\hline VS1 & $3(1-0-0-0-0-2)$ & $3(1-0-0-0-0-2)$ & VS1 & $4(0-1-1-0-0-1)$ & $4(0-1-1-0-0-2)$ \\
\hline VS2 & $4(1-0-1-0-0-2)$ & $4(1-0-1-0-0-2)$ & VS2 & $5(1-1-1-0-0-2)$ & $5(1-1-1-0-0-2)$ \\
\hline VS3 & $4(1-0-1-0-0-2)$ & $5(1-0-2-0-0-2)$ & VS3 & $4(0-1-1-0-0-2)$ & $4(0-1-1-0-0-2)$ \\
\hline VS4 & $6(1-1-2-0-0-2)$ & $9(1-3-3-0-0-2)$ & VS4 & $5(1-1-1-0-0-2)$ & $5(1-1-1-0-0-2)$ \\
\hline VS5 & $3(0-0-2-0-0-1)$ & $3(0-0-2-0-0-1)$ & VS5 & $7(1-1-2-1-0-2)$ & $8(2-1-2-1-0-2)$ \\
\hline MCS1 & $7(1-2-2-0-0-2)$ & $16(3-4-4-1-1-3)$ & VS6 & $4(0-1-1-0-0-2)$ & $9(2-2-3-0-0-2)$ \\
\hline MCS2 & $11(2-3-3-0-0-3)$ & $15(3-3-4-1-1-3)$ & MCS1 & $9(2-2-3-0-0-2)$ & $12(2-3-3-1-0-3)$ \\
\hline MCS3 & $10(2-3-2-0-0-3)$ & $12(2-3-3-1-0-3)$ & MCS2 & $9(1-2-3-1-0-2)$ & $10(1-3-3-1-0-2)$ \\
\hline MCS4 & $8(1-2-2-1-0-2)$ & $21(4-5-5-3-1-3)$ & MCS3 & $11(3-3-2-0-1-2)$ & $12(3-3-3-0-1-2)$ \\
\hline MCS5 & $9(2-3-2-0-0-2)$ & $14(2-3-3-1-0-3)$ & MCS4 & $12(3-3-4-0-0-2)$ & $16(4-4-4-1-0-3)$ \\
\hline MCS6 & $7(1-2-2-0-0-2)$ & $19(3-4-5-3-1-3)$ & MCS5 & $10(2-3-1-1-0-3)$ & $11(2-3-2-1-0-3)$ \\
\hline MCS7 & $6(1-2-2-0-0-1)$ & $21(3-5-6-3-1-3)$ & MCS6 & $12(2-3-3-2-0-2)$ & $15(3-4-3-2-0-3)$ \\
\hline MCS8 & $11(2-2-2-1-1-3)$ & $22(4-5-6-3-1-3)$ & MCS7 & $9(1-2-3-1-0-2)$ & $12(2-3-4-1-0-2)$ \\
\hline
\end{tabular}

VS, vegetative state; MCS, minimally conscious state; CRS-R, Coma Recovery Scale-Revised; tDCS, transcranial direct current stimulation.

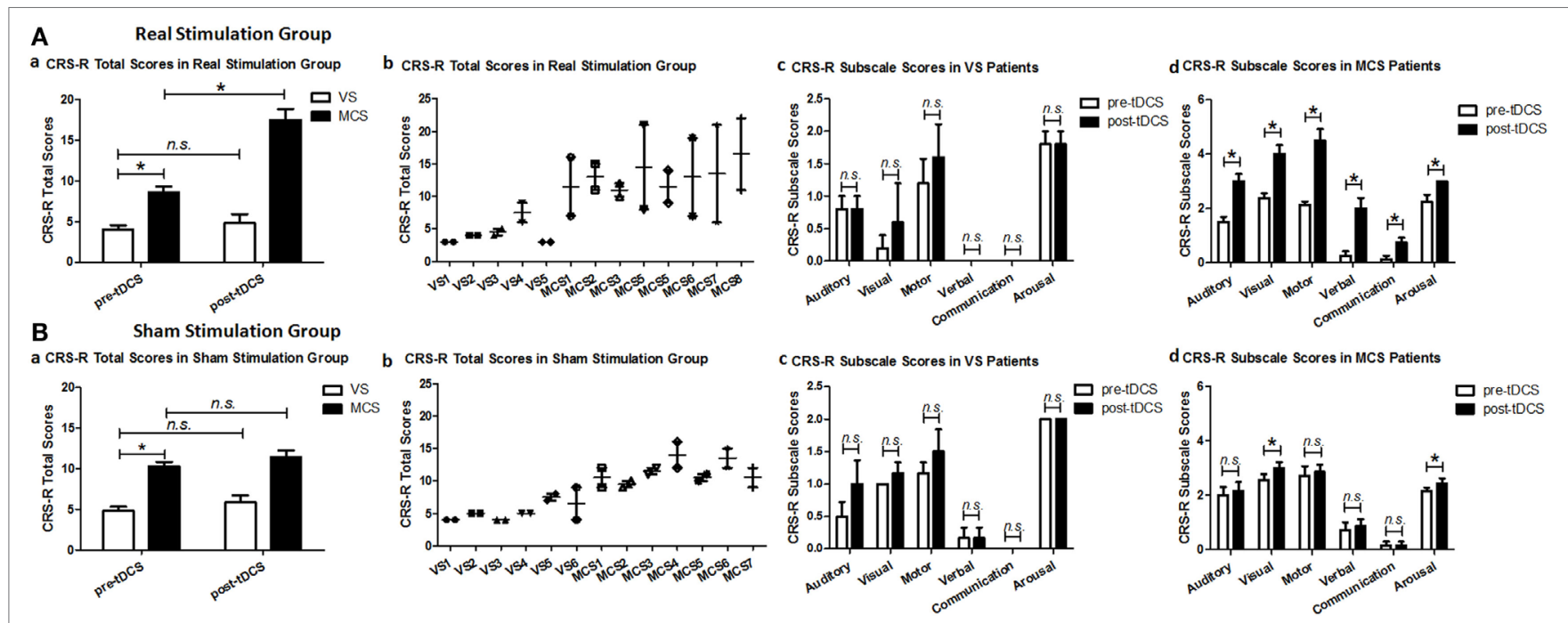

FIGURE 1 | Coma Recovery Scale-Revised (CRS-R) total scores and CRS-R subscale scores for the vegetative state (VS) and minimally conscious state (MCS) patients in (A) the real stimulation group and (B) the sham stimulation group at baseline (pre-tDCS) and after 20 sessions of transcranial direct current stimulation (tDCS) (post-tDCS). * $p<0.05$. Non-significant (n.s.): difference was not statistically significant.

\section{ERP Results}

In the real and sham stimulation groups, we compared the average latencies and amplitudes of P300 obtained using the midline electrodes ( $\mathrm{Fz}, \mathrm{CZ}$, and $\mathrm{Pz}$ ) at baseline (pre-tDCS) and after 20 sessions of tDCS (post-tDCS) in each group (VS and MCS), and the presence or absence of P300 between each group (VS and MCS) at baseline.

In our study, only one patient's ERP (VS2 in sham stimulation group) was recorded at the patient's bedside (both pre-tDCS and post-tDCS) instead of the ERP experiment room.

We have reported that a P300 wave was present in both TO and DO paradigms in 16 healthy subjects in a previous study (Figure S1 in Supplementary Material) (20). Figure 2 shows the grand averages of $\mathrm{P} 300$ at baseline and after tDCS in the VS and MCS patients at $\mathrm{Fz}, \mathrm{Cz}$, and $\mathrm{Pz}$ for the two paradigms in the real and sham stimulation groups. In the real and sham stimulation groups, there were no P300 waves in either paradigm before or after tDCS in the VS patients. The P300 topographies in the MCS patients showed that the two paradigms elicited a more pronounced frontal P300 effect (both at baseline and after tDCS) in the real and sham stimulation groups.

Regarding the P300 amplitudes, the Stimulation (real, sham $) \times$ Paradigms $($ TO, DO $) \times$ Time $($ pre-tDCS, post-tDCS $)$ repeated measures ANOVA revealed a main effect of Time $[F(1,18)=$ 9.25, $p=0.007$, and an interaction between Time and Stimulation $[F(1,18)=5.38, p=0.032]$. Post hoc comparison revealed that a significant increase in P300 amplitudes was observed in the MCS patients in the real stimulation group $[F(1,20)=14.55$, $p=0.001]$, but no significant difference was observed in the sham stimulation group $(F<1)$. Regarding the P300 latencies, a threeway repeated ANOVA revealed no significant main effect of Time $[F(1,18)=4.26, p=0.054]$, Stimulation $(F<1)$, or Paradigms 


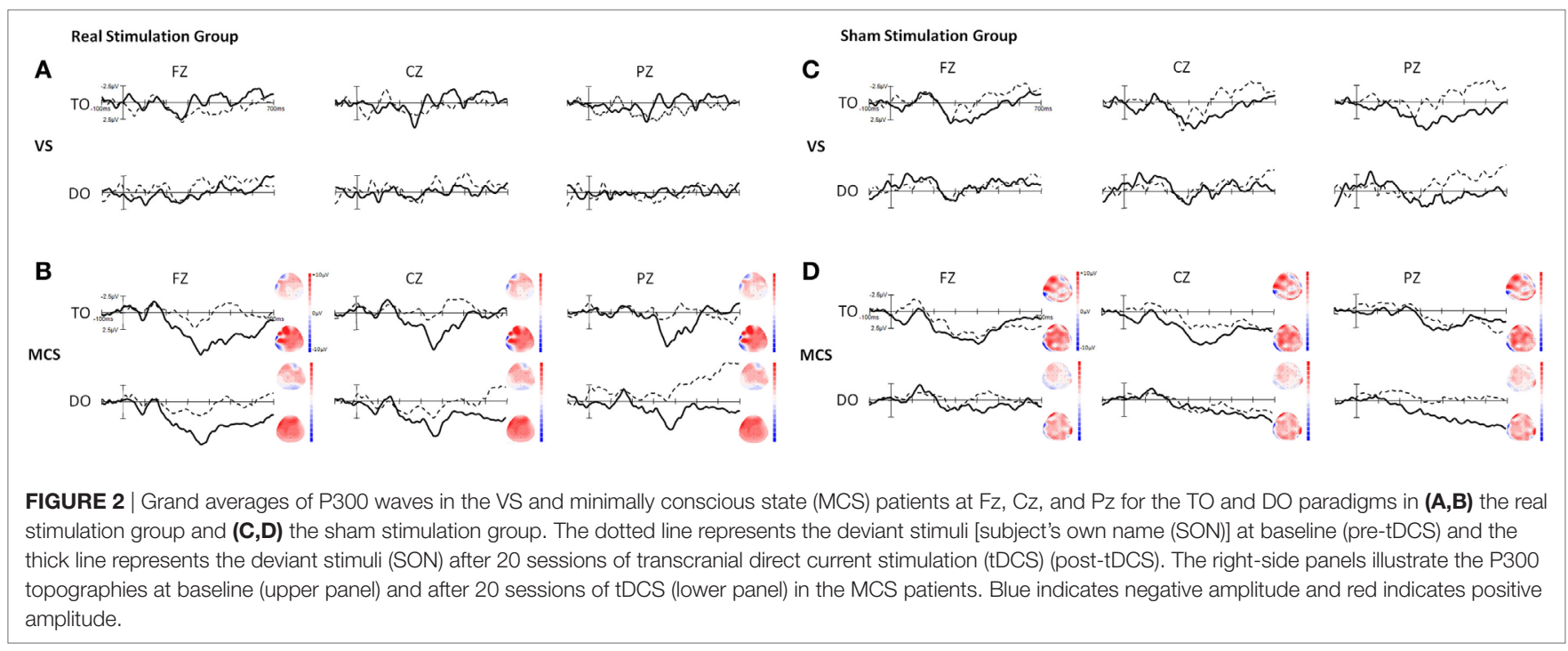

$(F<1)$. The results are shown in Tables 3 and $\mathbf{4}$ and Figure 3. From the inter-group analysis of ERP values at baseline, we observed significant differences in the presence of $\mathrm{P} 300$ waves for the TO paradigm $\left(\chi^{2}=5.923, p=0.032\right)$ and the DO paradigm $\left(\chi^{2}=6.964, p=0.021\right)$ between the VS and MCS patients in the real stimulation group, as well as for the TO paradigm $\left(\chi^{2}=6.741\right.$, $p=0.021)$ and the DO paradigm $\left(\chi^{2}=6.198, p=0.029\right)$ in the sham stimulation group according to Fisher's exact test (Table 5).

\section{DISCUSSION}

Previous studies have reported the effects of a single session and multiple sessions of tDCS on clinical improvement of patients with DOCs $(8,9,24)$, but this therapy is still far from becoming an established clinical practice. There is a pressing need to develop assessment methods to evaluate the treatment effects of tDCS in patients with DOCs, and these methods could also contribute to the understanding of the underlying mechanisms of tDCS. ERPs, mainly the P300 component, have been widely used to detect the electrophysiological correlates of cognitive capabilities, potentially reflecting residual levels of awareness in patients with DOCs $(25,26)$. In our study, the Fisher's exact test results demonstrate that the presence of $\mathrm{P} 300$ in response to the TO and DO paradigms could be used as a distinctive marker between VS and MCS patients at baseline. The results support the idea that the P300 wave in the oddball paradigms can be used to accurately characterize the level of cognitive preservation in patients with DOCs. However, the present ERP results were based on the analysis of group level rather than an individual participant level. Therefore, an integrated assessment of individual patient with DOCs using electrophysiological methods along with behavioral observation could refine the diagnostic and prognostic evaluation more accurately.

Probing for covert cognitive resources in patients with DOCs using electrophysiological methods requires special stimuli with an established probability of eliciting P300 responses. As demonstrated in the "cocktail party" phenomenon, SON has been frequently used to capture attention and evoke a powerful emotional reaction (27). Our study contrasted SON with SDN and recorded an auditory ERP pattern associated with increasing task complexity $(15,20,21)$. The grand average results revealed a larger P300 in response to SON after tDCS compared to before tDCS in MCS patients in the real stimulation group but not in VS patients. This confirms earlier findings that MCS patients have more residual cognitive resources than VS patients.

In our study, we used "passive" paradigms that are independent of the patient's collaboration and have been shown to have significantly fewer limitations than "active" paradigms, e.g., misunderstanding of task instructions by the patients. The ultimate goal when establishing an ERP task for patients with severe brain injury is not only to elicit cognitively mediated responses but also to not exceed the cognitive capacity of the patients. We believe that actively listening to pitch or counting target stimuli requires higher cognitive capacities compared to just listening. In addition, active ERP tasks require the patients not only to stay awake during the recording but also to understand the commands, be able to hold perceptual representations in their working memory, and complete the task (17). Although it was reported that passive oddball paradigms using the SON were known to elicit responses even during sleep (28), it could also help to detect changes in brain activity due to treatment, which contributed to understand the potential electrophysiological mechanism of tDCS.

The DLPFC plays a central integrative role in motor control and behavior, and it is an important component of the decisionmaking network (29-31). Studies have showed that as complexity or integration demands during action control increase, the DLPFC is increasingly associated with "top-down" cognitive control $(3,32,33)$. The P300 amplitude depends not only on the stimulus saliency but also on the participant's attentiveness $(34,35)$. Previous studies have reported that there is a variation in the P300 amplitude according to the amount of focal attention on discriminate stimuli. It can be speculated that the observed tDCSrelated consciousness improvements (as assessed by changes in the CRS-R score) are potentially related to improvements in 
TABLE 3 | Amplitude of P300 ( $\mu$ V) in VS and MCS groups in the TO and DO paradigms at baseline (pre-tDCS) and after 20 sessions of tDCS (post-tDCS).

\begin{tabular}{|c|c|c|c|c|c|c|c|c|c|c|c|c|c|c|c|c|c|c|c|c|c|c|c|c|c|}
\hline \multirow{3}{*}{$\begin{array}{l}\text { Real } \\
\text { stimulation }\end{array}$} & \multicolumn{6}{|c|}{ P300 amplitude in TO paradigm } & \multicolumn{6}{|c|}{ P300 amplitude in DO paradigms } & \multirow{3}{*}{$\begin{array}{l}\text { Sham } \\
\text { stimulation }\end{array}$} & \multicolumn{6}{|c|}{ P300 amplitude in TO paradigm } & \multicolumn{6}{|c|}{ P300 amplitude in DO paradigms } \\
\hline & \multicolumn{3}{|c|}{ Pre-tDCS } & \multicolumn{3}{|c|}{ Post-tDcs } & \multicolumn{3}{|c|}{ Pre-tDCs } & \multicolumn{3}{|c|}{ Post-tDCS } & & \multicolumn{3}{|c|}{ Pre-tDCS } & \multicolumn{3}{|c|}{ Post-tDCS } & \multicolumn{3}{|c|}{ Pre-tDCs } & \multicolumn{3}{|c|}{ Post-tDCS } \\
\hline & $\mathbf{F z}$ & $\mathrm{Cz}$ & $\mathbf{P z}$ & $\mathbf{F z}$ & $\mathrm{Cz}$ & $\mathbf{P z}$ & $\mathbf{F z}$ & $\mathrm{Cz}$ & $\mathbf{P z}$ & Fz & $\mathrm{Cz}$ & $\mathbf{P z}$ & & $\mathbf{F z}$ & $\mathrm{Cz}$ & $\mathbf{P z}$ & $z$ & $\mathrm{Cz}$ & z & $\mathrm{Fz}$ & $\mathrm{Cz}$ & $\mathbf{P z}$ & $\mathbf{F z}$ & $\mathrm{Cz}$ & $\mathbf{P z}$ \\
\hline ls1 & - & - & - & - & - & - & - & - & - & - & - & - & & - & - & - & - & - & - & - & - & 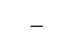 & - & - & - \\
\hline VS2 & - & - & - & - & - & - & - & - & - & - & - & - & & - & - & 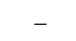 & - & - & - & - & - & - & - & - & - \\
\hline /S3 & - & - & - & - & - & - & - & - & - & 2.40 & 4.75 & 4.40 & & - & - & - & - & - & - & - & - & - & - & - & - \\
\hline VS4 & - & - & - & 10.45 & 4.62 & 1.70 & - & - & - & 8.11 & 4.10 & 1.05 & Vs & 1.30 & 2.89 & 4.81 & 3.93 & 4.01 & 2.48 & 12.05 & 11.27 & 4.69 & 2.69 & 4.20 & 3.72 \\
\hline VS5 & 3.73 & 3.38 & 3.71 & 0 & 0.88 & 2. & - & - & - & 1.16 & 1.69 & 2.20 & Vs & 8.85 & 9.14 & 0.47 & 7.01 & 7.47 & 7.03 & - & - & - & - & - & - \\
\hline MCS & 5.10 & 5.81 & 2.58 & 11.47 & 12.46 & 6.95 & 5.93 & 5.82 & 0.34 & 4.64 & 5.71 & 3.76 & Vs & - & - & - & 4.02 & 4.08 & 3.17 & - & - & - & - & - & - \\
\hline MCS2 & 0.71 & 2.01 & 2.45 & 2.14 & 3.05 & 2.66 & 2.10 & 3.59 & 0.85 & 4.41 & 7.40 & 5.82 & MCS1 & 11.42 & 6.12 & 2.33 & 5.49 & 2.12 & 0.24 & 2.10 & 3.59 & 0.85 & 7.53 & 6.57 & 4.68 \\
\hline MCS3 & 8.00 & 7.10 & 5.60 & 5.86 & 8.13 & 12.00 & 6.13 & 6.67 & 4.58 & 10.77 & 10.91 & 9.52 & MCS2 & 5.68 & 6.64 & 3.30 & 11.71 & 14.02 & 6.00 & 5.42 & 6.14 & 0 & 4.77 & 5.31 & 4.43 \\
\hline MCS4 & 4.03 & 3.47 & 1.98 & 3.73 & 4.25 & 3.76 & - & - & - & 4.25 & 8.36 & 3.72 & MCS3 & 8.68 & 10.61 & 10.44 & 0.83 & 6.56 & 2.70 & 3.45 & 5.85 & 4.90 & 2.19 & 7.17 & 9.04 \\
\hline MCS5 & 6.53 & 0.37 & 0 & 19.90 & 19.11 & 3.07 & 5.96 & 3.33 & 1.59 & 22.45 & 10.20 & 5.30 & MCS4 & 5.91 & 11.59 & 15.06 & 9.87 & 17.98 & 20.29 & 1.14 & 5.30 & 7.53 & 3.85 & 6.44 & 8.75 \\
\hline MCS6 & - & - & - & 4.21 & 2.25 & 1.80 & 1.32 & 3.67 & 4.70 & 7.03 & 5.24 & 4.84 & MCs5 & 0.83 & 3.36 & 13.00 & 0.64 & 9.66 & 13.61 & 0 & 11.00 & 8.35 & 2.76 & 4.81 & 5.19 \\
\hline MCS7 & 0 & 0.08 & 5.43 & 6.53 & 7.99 & 12.36 & 0.70 & 4.18 & 5.27 & 4.38 & 5.95 & 7.31 & MCS6 & 10.33 & 6.63 & 3.82 & 10.42 & 7.12 & 2.12 & 3.99 & 5.11 & 5.32 & 4.25 & 2.46 & 5.28 \\
\hline MCS8 & 10.73 & 11.32 & 9.62 & 4.75 & 5.26 & 4.06 & - & - & - & 4.21 & 4.31 & 4.91 & MCS7 & 8.45 & 2.75 & 1.14 & 8.26 & 1.71 & 0.54 & - & - & - & 8.68 & 8.23 & 7.88 \\
\hline
\end{tabular}

VS, vegetative state; MCS, minimally conscious state; TO, Tone-SON; DO, SDN-SON; SON, subject's own name; SDN, subject's derived name; tDCS, transcranial direct current stimulation.

TABLE 4 | Latency of P300 (ms) in VS and MCS groups in the TO and DO paradigms at baseline (pre-tDCS) and after 20 sessions of tDCS (post-tDCS).

\begin{tabular}{|c|c|c|c|c|c|c|c|c|c|c|c|c|c|c|c|c|c|c|c|c|c|c|c|c|c|}
\hline \multirow{3}{*}{$\begin{array}{l}\text { Real } \\
\text { Stimulation }\end{array}$} & \multicolumn{6}{|c|}{ P300 latency in TO paradigm } & \multicolumn{6}{|c|}{ P300 latency in DO paradigms } & \multirow{3}{*}{$\begin{array}{l}\text { Sham } \\
\text { stimulation }\end{array}$} & \multicolumn{6}{|c|}{ P300 latency in TO paradigm } & \multicolumn{6}{|c|}{ P300 latency in DO paradigms } \\
\hline & \multicolumn{3}{|c|}{ Pre-tDCs } & \multicolumn{3}{|c|}{ Post-tDcs } & \multicolumn{3}{|c|}{ Pre-tDcs } & \multicolumn{3}{|c|}{ Post-tDcs } & & \multicolumn{3}{|c|}{ Pre-tDcs } & \multicolumn{3}{|c|}{ Post-tDCS } & \multicolumn{3}{|c|}{ Pre-tDcs } & \multicolumn{3}{|c|}{ Post-tDcs } \\
\hline & Fz & $\mathrm{Cz}$ & $\mathbf{P z}$ & $\mathbf{F z}$ & $\mathrm{Cz}$ & $\mathbf{P z}$ & Fz & $\mathrm{Cz}$ & $\mathbf{P z}$ & $\mathrm{Fz}$ & $\mathrm{Cz}$ & $\mathbf{P z}$ & & $\mathbf{F z}$ & $\mathrm{Cz}$ & $\mathbf{P z}$ & $\mathbf{F z}$ & $\mathrm{Cz}$ & $\mathbf{P z}$ & $\mathbf{F z}$ & $\mathrm{Cz}$ & $\mathbf{P z}$ & Fz & $\mathrm{Cz}$ & $\mathbf{P z}$ \\
\hline /S1 & - & - & - & - & - & - & - & - & - & - & - & - & VS1 & - & - & - & - & - & - & - & - & - & - & - & - \\
\hline VS2 & - & - & - & - & - & - & - & - & - & - & - & - & Vs & - & - & - & - & - & - & - & - & - & - & - & - \\
\hline VS3 & - & - & - & - & - & - & - & - & - & 459 & 460 & 460 & Vs & - & - & - & - & - & - & - & - & - & - & - & - \\
\hline VS4 & - & - & - & 400 & 400 & 419 & - & - & - & 539 & 561 & 540 & VS4 & 320 & 365 & 365 & 368 & 367 & 367 & 406 & 407 & 408 & 410 & 437 & 437 \\
\hline VS5 & 414 & 415 & 416 & 499 & 500 & 503 & - & - & - & 428 & 432 & 453 & VS5 & 311 & 328 & 328 & 341 & 341 & 337 & - & - & - & - & - & - \\
\hline MCS1 & 407 & 369 & 367 & 368 & 365 & 365 & 385 & 383 & 382 & 411 & 376 & 376 & VS6 & - & - & - & 588 & 587 & 510 & - & - & - & - & - & - \\
\hline MCS2 & 672 & 671 & 672 & 500 & 493 & 490 & 570 & 543 & 546 & 539 & 562 & 563 & MCS1 & 464 & 505 & 484 & 493 & 498 & 507 & 570 & 543 & 547 & 451 & 430 & 428 \\
\hline MCS3 & 369 & 367 & 346 & 412 & 363 & 361 & 578 & 574 & 530 & 373 & 372 & 371 & MCS2 & 405 & 372 & 370 & 371 & 369 & 369 & 384 & 381 & 363 & 410 & 377 & 377 \\
\hline MCS4 & 574 & 572 & 573 & 376 & 381 & 380 & - & - & - & 553 & 554 & 553 & MCS3 & 680 & 662 & 655 & 658 & 685 & 659 & 699 & 620 & 641 & 699 & 699 & 699 \\
\hline MCS5 & 346 & 343 & 350 & 357 & 376 & 355 & 376 & 344 & 344 & 366 & 359 & 357 & MCS4 & 406 & 418 & 421 & 412 & 409 & 424 & 466 & 463 & 462 & 429 & 432 & 434 \\
\hline MCS6 & - & - & - & 564 & 564 & 515 & 388 & 400 & 433 & 463 & 454 & 446 & MCS5 & 688 & 684 & 687 & 699 & 687 & 690 & 616 & 690 & 688 & 690 & 690 & 689 \\
\hline MCS7 & 500 & 545 & 545 & 477 & 475 & 474 & 554 & 556 & 559 & 349 & 372 & 388 & MCS6 & 316 & 320 & 329 & 316 & 321 & 324 & 307 & 303 & 307 & 305 & 319 & 313 \\
\hline MCS8 & 582 & 527 & 528 & 552 & 552 & 500 & - & - & - & 630 & 629 & 627 & MCS7 & 585 & 597 & 599 & 424 & 400 & 400 & - & - & - & 678 & 607 & 608 \\
\hline
\end{tabular}

VS, vegetative state; MCS, minimally conscious state; TO, Tone-SON; DO, SDN-SON; SON, subject's own name; SDN, subject's derived name; tDCS, transcranial direct current stimulation. 


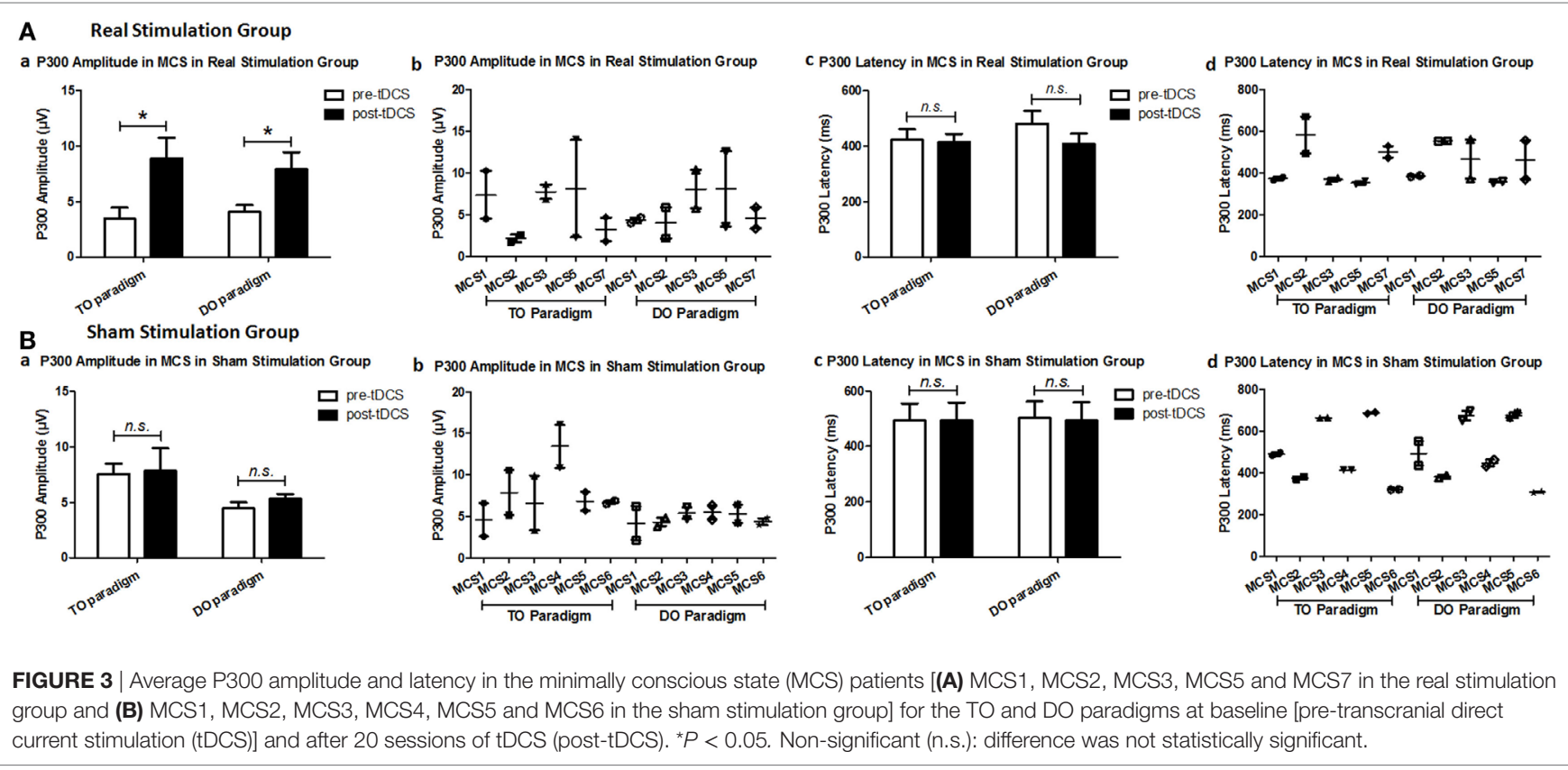

TABLE 5 | Presence or absence of P300 in VS and MCS groups in the TO and DO paradigms at baseline.

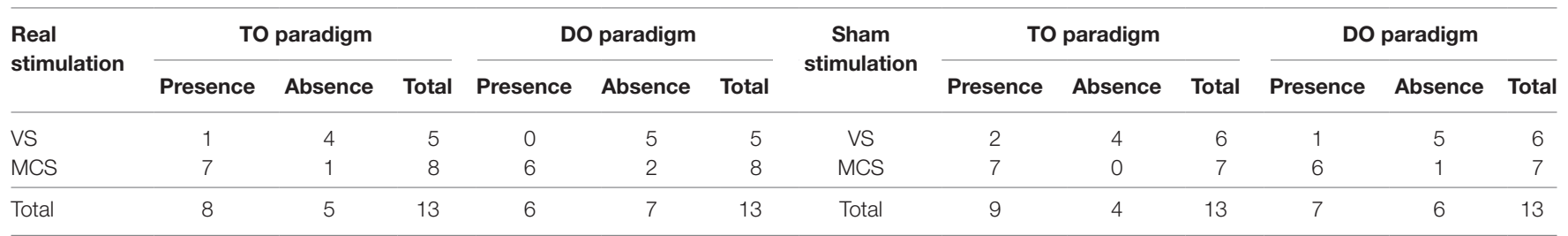

VS, vegetative state; MCS, minimally conscious state; TO, Tone-SON; DO, SDN-SON; SON, subject's own name; SDN, subject's derived name.

attention resource allocation (as reflected by the $\mathrm{P} 300$ amplitude). In contrast, a lack of P300 amplitude modulation might reflect an impairment of processing requiring higher attention. It has been reported that $\mathrm{P} 300$ latency can be used as an objective index of stimulus evaluation time and it is sensitive to task complexity. Therefore, a more complex stimulus can lead to a delay in orientation and engagement with new stimuli (16). In our current study, the P300 latency tended to increase with enhancement of stimulus complexity in the MCS group, but there was no significant difference at baseline. A study by Cavinato et al. showed that, in MCS patients, P300 latency was modulated at different levels of stimulus complexity (16). The inconsistency between our results and those of the study by Cavinato et al. might be due in part to the small sample size and the clinical heterogeneity of the patients with DOCs in our study.

Our study showed that there was a statistically significant improvement in the clinical condition of the MCS patients, but not in the VS patients in the real stimulation group. These findings are in line with those of a previous study that demonstrated that out of three patients in prolonged MCSs, all showed mild behavioral improvements after five daily stimulation sessions, but none of the seven VS patients showed behavioral improvements at the end of the stimulation protocol (8). In another recent study of patients with prolonged DOCs (22), repeated
tDCS did not lead to a remarkable short-term clinical improvement or to EEG effects. The major difference between the two results may be related to the participants' postinjury durations. A study demonstrated that consciousness improvement is possible in patients with DOCs despite several months in VS or MCS, suggesting that there remains potential for behavioral improvement even for severely affected patients with DOCs (36). Therefore, we did not exclude the VS or MCS patients for greater than 12 months. In addition, although pediatric brain recovery may differ from adult brain recovery, we did not specially focus on the relevance of ages in patients because of the relatively small sample size in our current study. Statistical analysis showed that there was no statistical difference between the group ages. However, the effect of injury duration and age will be the focus of our following studies.

Recently, an increasing number of studies have explored the effects of non-invasive brain stimulation, including TMS and tDCS, on improvements in consciousness in patients with DOCs (8-10, 37-39). However, in practice, tDCS is easier to use, requires a smaller device, and is less expensive (40) than TMS. In particular, tDCS could be used early in DOC rehabilitation programs due to its safety. The evaluation tools used to assess therapeutic efficacy in patients with DOCs include neurophysiological and functional neuroimaging techniques. However, despite the unquestionable 
value of magnetoencephalogram, positron emission tomography, and fMRI studies, these procedures cannot be performed at the patient's bedside. In such cases, the use of electrophysiological recordings is more practical and appropriate.

Although the results are exciting, some questions remain to be addressed in future studies. First, the main limitations of this study are the small sample size and lack of long-term follow-up, and we only presented a group analysis but not an evaluation of the individual response to tDCS. Thus, future investigations should optimize the tDCS parameters in an individual analysis in a larger and more representative sample of patients with DOCs involving longitudinal follow-ups. Second, a methodological limitation of our study is the absence of MRI-based mapping to identify the location of the stimulated area. The large structural lesions in patients with severe brain injury may have potential influence on the effect of tDCS (41). Recent studies demonstrated that the response to tDCS in MCS patients might be related to the residual metabolic activity in brain areas including the left DLPFC (42), as well as high connectivity with regions belonging to extrinsic control network (43). Further studies should employ an individualized tDCS protocol based on patient-tailored brain structure. Third, the difference in stimulus intensity in a number of patients ( $1 \mathrm{~mA}$ instead of $2 \mathrm{~mA}$ because of the craniotomy) might add to the variability of the small sample in our study. Future studies should deeply investigate whether or how such change in stimulus intensity had any effect. Fourth, since neurophysiological and functional neuroimaging techniques explore different aspects and consequently provide different information, these methods should be applied together in future studies to provide a broader and more holistic evaluation of therapeutic efficacy in patients with DOCs.

\section{CONCLUSION}

To the best of our knowledge, this is the first study to provide behavioral and ERP evidence of the effect of tDCS in patients with DOCs. In summary, our study revealed that repeated anodal tDCS of the left DLPFC may produce clinically significant improvements and modulate the P300 amplitude in MCS patients. The study also

\section{REFERENCES}

1. Multi-Society Task Force on PVS. Medical aspects of the persistent vegetative state (1). N Engl J Med (1994) 330:1499-508. doi:10.1056/NEJM199405263302107

2. Giacino JT, Ashwal S, Childs N, Cranford R, Jennett B, Katz DI, et al. The minimally conscious state: definition and diagnostic criteria. Neurology (2002) 58:349-53. doi:10.1212/WNL.58.3.349

3. Miller EK, Cohen JD. An integrative theory of prefrontal cortex function. Annu Rev Neurosci (2001) 24:167-202. doi:10.1146/annurev.neuro.24.1.167

4. Lafontaine MP, Theoret H, Gosselin F, Lippe S. Transcranial direct current stimulation of the dorsolateral prefrontal cortex modulates repetition suppression to unfamiliar faces: an ERP study. PLoS One (2013) 8:e81721. doi:10.1371/journal.pone.0081721

5. Vanderhasselt MA, De Raedt R, Brunoni AR, Campanha C, Baeken C, Remue J, et al. tDCS over the left prefrontal cortex enhances cognitive control for positive affective stimuli. PLoS One (2013) 8:e62219. doi:10.1371/journal. pone.0062219

6. Brunoni AR, Vanderhasselt MA. Working memory improvement with non-invasive brain stimulation of the dorsolateral prefrontal cortex: a highlighted the need to associate behavioral evaluation results with neurophysiological results in order to achieve a more objective and accurate assessment of patients with DOCs. Our results demonstrate that ERP recording might serve as an alternative assessment tool for evaluating the effectiveness of DOC treatments and supported the use of $\mathrm{tDCS}$ and ERP recording in clinical practice.

\section{ETHICS STATEMENT}

Written informed consents were acquired from all the patients' families or caregivers. The study was conducted according to the Declaration of Helsinki, and the ethics approval was provided by the ethics committee of the hospital.

\section{AUTHOR CONTRIBUTIONS}

Full access to all the data in the study and responsibility for the integrity of the data and the accuracy of the data analysis: YZ. Study concept and design; acquisition, analysis, or interpretation of data: YZ. Drafting of the manuscript: YZ. Critical revision of the manuscript for important intellectual content: all authors. Statistical analysis: YZ. Obtained funding: WS. Administrative, technical, or material support: GS and RL. Study supervision: JD, $\mathrm{SH}$, and WS.

\section{FUNDING}

This work was supported by the National Natural Science Foundation of China (grant numbers 81371194) in the data collection and analysis.

\section{SUPPLEMENTARY MATERIAL}

The Supplementary Material for this article can be found online at http://www.frontiersin.org/article/10.3389/fneur.2017.00620/ full\#supplementary-material.

FIGURE S1 | Grand averages of P300 waves in 16 healthy subjects at Fz, Cz, and $\mathrm{Pz}$ in the TO and DO paradigms. The thick line represents the deviant stimuli (subject's own name). systematic review and meta-analysis. Brain Cogn (2014) 86:1-9. doi:10.1016/j. bandc.2014.01.008

7. Nelson JT, Mckinley RA, Golob EJ, Warm JS, Parasuraman R. Enhancing vigilance in operators with prefrontal cortex transcranial direct current stimulation (tDCS). Neuroimage (2014) 85(Pt 3):909-17. doi:10.1016/j. neuroimage.2012.11.061

8. Angelakis E, Liouta E, Andreadis N, Korfias S, Ktonas P, Stranjalis G, et al. Transcranial direct current stimulation effects in disorders of consciousness. Arch Phys Med Rehabil (2014) 95:283-9. doi:10.1016/j.apmr.2013.09.002

9. Thibaut A, Bruno MA, Ledoux D, Demertzi A, Laureys S. tDCS in patients with disorders of consciousness: sham-controlled randomized double-blind study. Neurology (2014) 82:1112-8. doi:10.1212/WNL.0000000000000260

10. Thibaut A, Wannez S, Donneau AF, Chatelle C, Gosseries O, Bruno MA, et al. Controlled clinical trial of repeated prefrontal tDCS in patients with chronic minimally conscious state. Brain Inj (2017) 31:466-74. doi:10.1080/02699052. 2016.1274776

11. Bai Y, Xia X, Kang J, Yang Y, He J, Li X. TDCS modulates cortical excitability in patients with disorders of consciousness. Neuroimage Clin (2017) 15:702-9. doi:10.1016/j.nicl.2017.01.025 
12. Andrews K, Murphy L, Munday R, Littlewood C. Misdiagnosis of the vegetative state: retrospective study in a rehabilitation unit. BMJ (1996) 313:13-6. doi:10.1136/bmj.313.7048.13

13. Polich J. Updating P300: an integrative theory of P3a and P3b. Clin Neurophysiol (2007) 118:2128-48. doi:10.1016/j.clinph.2007.04.019

14. Morlet D, Fischer C. MMN and novelty P3 in coma and other altered states of consciousness: a review. Brain Topogr (2014) 27:467-79. doi:10.1007/ s10548-013-0335-5

15. Li R, Song WQ, Du JB, Huo S, Shan GX. Connecting the P300 to the diagnosis and prognosis of unconscious patients. Neural Regen Res (2015) 10:473-80. doi:10.4103/1673-5374.153699

16. Cavinato M, Volpato C, Silvoni S, Sacchetto M, Merico A, Piccione F. Eventrelated brain potential modulation in patients with severe brain damage. Clin Neurophysiol (2011) 122:719-24. doi:10.1016/j.clinph.2010.08.024

17. Hauger SL, Schnakers C, Andersson S, Becker F, Moberget T, Giacino JT, et al. Neurophysiological indicators of residual cognitive capacity in the minimally conscious state. Behav Neurol (2015) 2015:145913. doi:10.1155/ 2015/145913

18. Schnakers C, Giacino JT, Lovstad M, Habbal D, Boly M, Di H, et al. Preserved covert cognition in noncommunicative patients with severe brain injury? Neurorehabil Neural Repair (2015) 29:308-17. doi:10.1177/ 1545968314547767

19. Holeckova I, Fischer C, Giard MH, Delpuech C, Morlet D. Brain responses to a subject's own name uttered by a familiar voice. Brain Res (2006) 1082:142-52. doi:10.1016/j.brainres.2006.01.089

20. Li R, Song W, Du J, Huo S, Shan G. Electrophysiological correlates of processing subject's own name. Neuroreport (2015) 26:937-44. doi:10.1097/ WNR.0000000000000437

21. Zhang Y, Li R, Du J, Huo S, Hao J, Song W. Coherence in P300 as a predictor for the recovery from disorders of consciousness. Neurosci Lett (2017) 653:332-6. doi:10.1016/j.neulet.2017.06.013

22. Estraneo A, Pascarella A, Moretta P, Masotta O, Fiorenza S, Chirico G, et al. Repeated transcranial direct current stimulation in prolonged disorders of consciousness: a double-blind cross-over study. JNeurol Sci (2017) 375:464-70. doi:10.1016/j.jns.2017.02.036

23. Semlitsch HV, Anderer P, Schuster P, Presslich O. A solution for reliable and valid reduction of ocular artifacts, applied to the P300 ERP. Psychophysiology (1986) 23:695-703. doi:10.1111/j.1469-8986.1986.tb00696.x

24. Zhang Y, Song W. Transcranial direct current stimulation in disorders of consciousness: a review. Int J Neurosci (2017):1-7. doi:10.1080/00207454. 2017.1357552

25. Real RG, Veser S, Erlbeck H, Risetti M, Vogel D, Muller F, et al. Information processing in patients in vegetative and minimally conscious states. Clin Neurophysiol (2016) 127:1395-402. doi:10.1016/j.clinph.2015.07.020

26. Ragazzoni A, Cincotta M, Giovannelli F, Cruse D, Young GB, Miniussi C, et al. Clinical neurophysiology of prolonged disorders of consciousness: from diagnostic stimulation to therapeutic neuromodulation. Clin Neurophysiol (2017) 128:1629-46. doi:10.1016/j.clinph.2017.06.037

27. Perrin F, Castro M, Tillmann B, Luaute J. Promoting the use of personally relevant stimuli for investigating patients with disorders of consciousness. Front Psychol (2015) 6:1102. doi:10.3389/fpsyg.2015.01102

28. Perrin F, Garcia-Larrea L, Mauguiere F, Bastuji H. A differential brain response to the subject's own name persists during sleep. Clin Neurophysiol (1999) 110:2153-64. doi:10.1016/S1388-2457(99)00177-7

29. Heekeren HR, Marrett S, Ruff DA, Bandettini PA, Ungerleider LG. Involvement of human left dorsolateral prefrontal cortex in perceptual decision making is independent of response modality. Proc Natl Acad Sci U S A (2006) 103:10023-8. doi:10.1073/pnas.0603949103
30. Metuki N, Sela T, Lavidor M. Enhancing cognitive control components of insight problems solving by anodal tDCS of the left dorsolateral prefrontal cortex. Brain Stimul (2012) 5:110-5. doi:10.1016/j.brs.2012.03.002

31. Enriquez-Geppert S, Huster RJ, Herrmann CS. Boosting brain functions: improving executive functions with behavioral training, neurostimulation, and neurofeedback. Int JPsychophysiol (2013) 88:1-16. doi:10.1016/j. ijpsycho.2013.02.001

32. Faber M, Vanneste S, Fregni F, De Ridder D. Top down prefrontal affective modulation of tinnitus with multiple sessions of tDCS of dorsolateral prefrontal cortex. Brain Stimul (2012) 5:492-8. doi:10.1016/j.brs.2011.09.003

33. Cieslik EC, Zilles K, Caspers S, Roski C, Kellermann TS, Jakobs O, et al. Is there "one" DLPFC in cognitive action control? Evidence for heterogeneity from co-activation-based parcellation. Cereb Cortex (2013) 23:2677-89. doi:10.1093/cercor/bhs256

34. Pritchard WS. Psychophysiology of P300. Psychol Bull (1981) 89:506-40. doi:10.1037/0033-2909.89.3.506

35. Johnson R Jr. A triarchic model of P300 amplitude. Psychophysiology (1986) 23:367-84. doi:10.1111/j.1469-8986.1986.tb00649.x

36. Howell K, Grill E, Klein AM, Straube A, Bender A. Rehabilitation outcome of anoxic-ischaemic encephalopathy survivors with prolonged disorders of consciousness. Resuscitation (2013) 84:1409-15. doi:10.1016/j.resuscitation. 2013.05.015

37. Cincotta M, Giovannelli F, Chiaramonti R, Bianco G, Godone M, Battista D, et al. No effects of $20 \mathrm{~Hz}-\mathrm{rTMS}$ of the primary motor cortex in vegetative state: a randomised, sham-controlled study. Cortex (2015) 71:368-76. doi:10.1016/j. cortex.2015.07.027

38. Naro A, Russo M, Leo A, Bramanti P, Quartarone A, Calabro RS. A single session of repetitive transcranial magnetic stimulation over the dorsolateral prefrontal cortex in patients with unresponsive wakefulness syndrome: preliminary results. Neurorehabil Neural Repair (2015) 29:603-13. doi:10.1177/1545968314562114

39. Bai Y, Xia X, Kang J, Yin X, Yang Y, He J, et al. Evaluating the effect of repetitive transcranial magnetic stimulation on disorders of consciousness by using TMS-EEG. Front Neurosci (2016) 10:473. doi:10.3389/fnins.2016.00473

40. Floel A. tDCS-enhanced motor and cognitive function in neurological diseases. Neuroimage (2014) 85(Pt 3):934-47. doi:10.1016/j.neuroimage.2013.05.098

41. Minjoli S, Saturnino GB, Blicher JU, Stagg CJ, Siebner HR, Antunes A, et al. The impact of large structural brain changes in chronic stroke patients on the electric field caused by transcranial brain stimulation. Neuroimage Clin (2017) 15:106-17. doi:10.1016/j.nicl.2017.04.014

42. Thibaut A, Di Perri C, Chatelle C, Bruno MA, Bahri MA, Wannez S, et al. Clinical response to tDCS depends on residual brain metabolism and grey matter integrity in patients with minimally conscious state. Brain Stimul (2015) 8:1116-23. doi:10.1016/j.brs.2015.07.024

43. Cavaliere C, Aiello M, Di Perri C, Amico E, Martial C, Thibaut A, et al. Functional connectivity substrates for tDCS response in minimally conscious state patients. Front Cell Neurosci (2016) 10:257. doi:10.3389/fncel.2016.00257

Conflict of Interest Statement: The authors declare that the research was conducted in the absence of any commercial or financial relationships that could be construed as a potential conflict of interest.

Copyright (C) 2017 Zhang, Song, Du, Huo, Shan and Li. This is an open-access article distributed under the terms of the Creative Commons Attribution License (CC BY). The use, distribution or reproduction in other forums is permitted, provided the original author(s) or licensor are credited and that the original publication in this journal is cited, in accordance with accepted academic practice. No use, distribution or reproduction is permitted which does not comply with these terms. 